Classification

Physics Abstracts

$43.30-43.85-47.80$

\title{
Visualisation par moyens acoustiques de la dynamique sédimentaire autour d'un obstacle sur fond marin $(*)$
}

\author{
J. Bresson, F. X. de Contencin, C. Chaussavoine et J. P. Longuemard \\ C.R.S.M.P. Université de Perpignan, 66025 Perpignan, France
}

(Reçu le 11 mai 1983, révisé le 21 juin, accepté le 27 juin 1983)

\begin{abstract}
Résumé. - Nous décrivons un appareillage permettant de restituer la topographie du fond marin au voisinage d'un objet déposé sur du sable par petite profondeur. Basé sur des mesures acoustiques, ce dispositif permet le tracé de « cartes » à intervalles de temps réguliers et par comparaison d'évaluer le transit sédimentaire autour de l'objet en fonction des houles et des marées.

Après une description précise du principe de mesure de l'appareil lui-même et de ses performances, les auteurs fournissent quelques résultats obtenus sur un site côtier de l'Océan Atlantique.
\end{abstract}

\begin{abstract}
An apparatus to survey on the sediments surface, is described. Based on acoustic measurements, the system allows sea-bed mapping at regular time intervals and comparison of successive maps to estimate sediment transport around the object by swell and tides.

After a complete description of the principles, operation and performance of the acoustic system, results obtained along the European Atlantic coast are presented.
\end{abstract}

\section{Introduction.}

Le comportement d'un fond marin résulte des conditions hydrodynamiques, des caractéristiques des matériaux qui le constituent et de sa topographie. La présence d'un objet posé sur ce dernier détruit son équilibre et entraîne une modification de son état.

Un objet posé sur un fond cohésif a tendance à s'enfoncer par gravité, par contre s'il est posé sur un fond sableux, non cohésif, sa présence perturbe les conditions hydrodynamiques et entraîne une modification de la dynamique sédimentaire donc de la topographie autour de l'obstacle.

Des études expérimentales en canal ont permis d'obtenir des résultats qualitatifs sur les phénomènes physiques qui entrent en jeu. Cependant, il est délicat de transposer ces résultats en milieu naturel par suite des difficultés qu'il y a à respecter les échelles de similitudes géométrique et dynamique. On se propose donc d'étudier ce phénomène complexe sur un site naturel en vraie grandeur.

Les problèmes à résoudre dans le cadre de cette

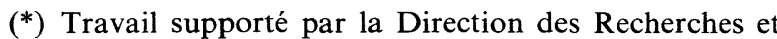
des Etudes Techniques. étude sont multiples et concernent deux pôles d'intérêts :

- l'hydrodynamique et les conditions d'écoulement autour d'un obstacle.

- la conception d'un appareil susceptible d'acquérir les conditions nécessaires pour quantifier l'évolution du fond.

Ce dernier point est plus particulièrement développé ici. Un procédé basé sur l'emploi des ultra-sons semble particulièrement bien adapté pour ce genre d'étude.

\section{Description de l'expérience.}

Une expérience globale sur site nécessite la mise en œuvre de différents appareils de mesure conditionnés par les contraintes d'essais et par le but à obtenir (modélisation de l'affouillement). Seule la détermination de l'affouillement présente une originalité. 2.1 CONDITIONS EXPÉRIMENTALES. - L'obstacle est constitué par un cylindre de $0,6 \mathrm{~m}$ de diamètre et $2 \mathrm{~m}$ de long, d'un poids apparent dans l'eau d'environ $700 \mathrm{~N}$, posé selon une génératrice sur un fond sableux par petites profondeurs $(10$ à $30 \mathrm{~m})$. Il est soumis à l'action conjuguée des courants de houle et de marée. Des essais préliminaires effectués en bassin ont 
montré que la zone notablement concernée par les perturbations de l'écoulement provoquées par l'obstacle correspond à un rectangle. Il a été décidé, compte tenu de ces résultats expérimentaux, d'explorer une zone de $2 \mathrm{~m}$ sur $4 \mathrm{~m}$ centrée sur l'objet et d'en faire périodiquement un relevé topographique.

La houle provoque une remise en suspension des particules. Il est logique de choisir une méthode acoustique plutôt qu'optique pour quantifier le volume du charriage sédimentaire. En effet, les ondes lumineuses ne permettraient pas dans ce cas d'obtenir des photographies de la surface du fond à étudier (diffusion des ondes autour des grains de sable).

Des mesures corrélatives de paramètres hydrodynamiques (courant, houle) permettront d'établir des relations entre les conditions d'écoulement et la dynamique sédimentaire.

2.2 DESCRIPTION SOMMAIRE DU MATÉRIEL DE CONTRÔLE ULTRA-SONORE. - La zone à contrôler est encadrée par un portique disposé de telle sorte que sa présence ne perturbe pas les conditions hydrodynamiques au voisinage du fond. Ce dernier, solidement ancré en dehors de la zone d'exploration (Fig. 1), supporte

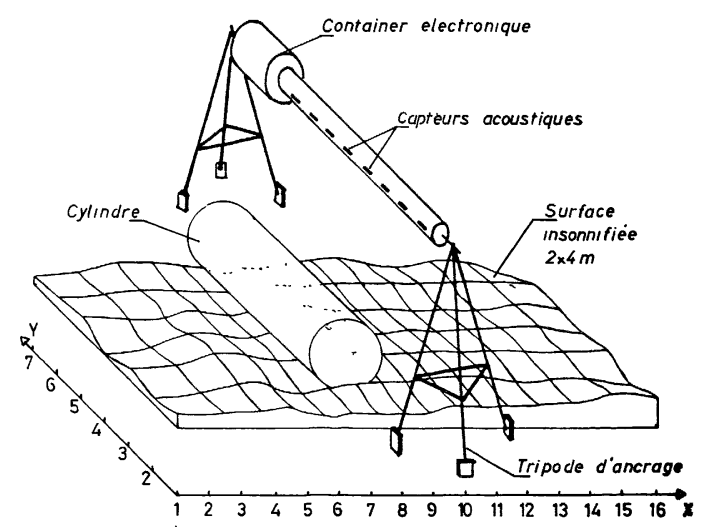

Fig. 1. - Schéma de l'appareillage.

[Schematic view of apparatus.]

un tube d'axe horizontal doté de sondes acoustiques situé à $2 \mathrm{~m}$ au-dessus du fond. Un caisson étanche monté en bout d'axe contient le dispositif électronique de commande, d'enregistrement et un moteur de commande de rotation du tube autour de son axe en 16 positions. Le principe de conception découle de la surface à contrôler (nombre d'incréments relativement facile à obtenir avec une électronique logique) et conduit à une ouverture de lobe de rayonnement à $3 \mathrm{~dB}$ de 6 degrés.

L'obtention du contrôle complet de la surface soumise à de grandes perturbations est assurée par 8 émetteurs-récepteurs qui agissent suivant 8 lignes perpendiculaires à l'axe de rotation de leur support. Une mesure de temps est effectuée à chaque position angulaire du tube pour chaque capteur. La zone à explorer est donc quadrillée à partir de 128 points de mesure $(16 \times 8)$.

Chacun d'entre eux conduit à un relevé de la hauteur relative de la surface élémentaire entourant un point. Un cycle complet permet l'acquisition d'un relevé topographique de la zone contrôlée et de dresser une carte du fond au voisinage de l'obstacle. La comparaison de plusieurs cartes successives est nécessaire pour effectuer le calcul du transit sédimentaire en quantité et en direction.

\section{Dispositif de mesure et de commande.}

3.1 PRINCIPE DE LA MESURE. - Les expériences se dérouleront dans un milieu turbulent avec une perpétuelle remise en suspension des grains constitutifs du fond marin.

Ce milieu opaque à une stimulation lumineuse, sera transparent à une excitation acoustique tant que l'absorption par diffusion de l'énergie acoustique restera faible. La longueur d'onde de la vibration devra être grande devant les dimensions des grains de sable [1].

La mesure acoustique pleinement justifiée d'un point de vue physique présente également un autre avantage : la mesure quantitative des phénomènes étudiés. Le principe de fonctionnement de l'appareil consiste à émettre une vibration acoustique dans le milieu ambiant en direction du fond et à déterminer la durée de parcours de l'onde après rétrodiffusion sur le sable.

3. 2 DÉFINITION DE LA FRÉQUENCE DE TRAVAIL ET DES SONDES ACOUSTIQUES. - La fréquence retenue résultera d'un compromis entre la résolution et la portée acoustique de l'appareillage.

L'énergie émise est confinée dans un angle solide qui dépend du diamètre des sondes et de la longueur d'onde de la vibration acoustique. L'ouverture du lobe de rayonnement pour un émetteur circulaire, est définie par [2] :

$$
\sin \theta_{3}=1,46 \frac{\lambda}{\pi D}
$$

où :

$\theta_{3}=1 / 2$ angle d'ouverture à $3 \mathrm{~dB}$,

$D=$ diamètre de la face émettrice,

$\lambda=$ longueur d'onde.

Une ouverture du lobe principal de $6^{\circ}$ pour chaque transducteur est nécessaire pour assurer une couverture totale de la zone étudiée autour de l'obstacle. Le diamètre de la sonde dépendra de ce fait de la fréquence ultra-sonore dont le choix est conditionné par :

1) l'absorption dans le milieu turbulent qui doit être le plus faible possible, ce qui impose une fréquence basse, 
2) le niveau réverbéré par le fond $R_{\mathrm{F}}$ qui croît avec la fréquence,

3) la précision de la mesure, en effet une onde de haute fréquence autorise l'utilisation de trains d'ondes de courte durée, ce qui améliore la définition, donc le rapport signal sur bruit de fond du système,

4) des critères techniques qui sont :

- l'encombrement des sondes, le diamètre des sondes étant inversement proportionnel à la fréquence pour une ouverture de lobe donnée,

- la nécessité de travailler en champ lointain, c'est-à-dire au-delà d'une certaine distance $r\left(r>D^{2} / \lambda\right)$ du transducteur qui nous est imposée par les conditions expérimentales.

Une onde acoustique d'intensité $I_{0}$ émise à la distance $r$ du point de rétrodiffusion provoque en retour un écho dont le niveau est défini par la relation ci-dessous :

$$
I_{r}=\frac{I_{0}}{r^{4}} \mathcal{R}_{\mathrm{F}} \exp (-2 \alpha r) \cdot A\left(\tau, \theta_{3}, \phi\right)
$$

Dans cette équation :

$\alpha \quad$ : représente l'atténuation en fonction de la fréquence $(F)$ et est proportionnel à $F^{2}[1,3]$

$A\left(\tau, \theta_{3}, \phi\right)$ : traduit l'importance de la surface soumise aux ultrasons et par voie de conséquence agit sur le niveau rétrodiffusé. $\mathrm{Ce}$ dernier varie donc avec l'angle d'incidence $\phi$, l'ouverture du lobe $\theta_{3}$, la durée de l'impulsion émise $\tau$ et la célérité des ondes dans l'eau $C$. Il est défini par la relation ci-dessous :

$$
A\left(\tau, \theta_{3}, \phi\right)=\frac{\theta_{3} r C \tau}{\sin \phi}
$$

$R_{\mathrm{F}}=10 \log R:$ représente l'index de réverbération de fond.

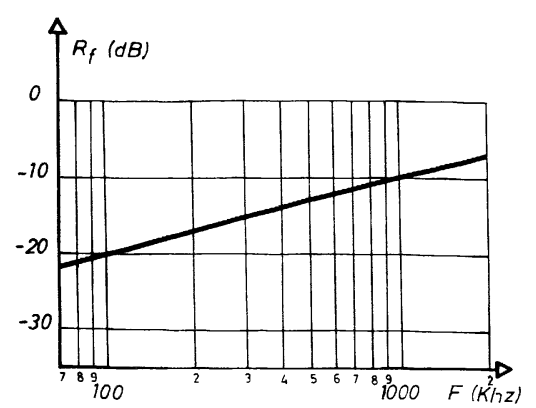

Fig. 2. - Index de réverbération du fond en fonction de la fréquence.

[Bottom backscattering coefficient versus frequency.]
L'équation générale susceptible de fournir la valeur de niveau reçu $L_{(\mathrm{dB})}$ en fonction du niveau émis $S_{(\mathrm{dB})}$ exprimé sous forme logarithmique est :

$$
L=S-40 \log r-2 \dot{\alpha} r+R_{\mathrm{F}}+10 \log A .
$$

Pratiquement, on peut écrire en tenant compte de la relation 3 :

$$
\begin{aligned}
L-S=-2 \alpha r- & 30 \log r+R_{\mathrm{F}}+10 \log \theta_{3}+ \\
& +10 \log C \tau-10 \log \sin \phi .
\end{aligned}
$$

Pour un lobe de rayonnement constant, $C$ et $\tau$ étant fixés et $\sin \phi$ compris entre 0 et $\pi / 2$, nous pouvons écrire que :

$$
L-S=-2 \alpha r+R_{\mathrm{F}}+\text { Cte }
$$

où seuls $\alpha$ et $R_{\mathrm{F}}$ influeront sur le facteur $L-S$.

Des études théoriques et des résultats expérimentaux obtenus par plusieurs auteurs [5-10] montrent que la réverbération $\mathrm{du}$ fond dépend du comportement acoustique des deux milieux (eau-sédiment), de la rugosité du substrat, de la longueur d'onde et de l'angle d'incidence. La valeur de $R_{\mathrm{F}}$ varie largement avec la rugosité du fond donc avec sa granulométrie. Les sables souvent soumis à des courants de houle présentent une hétérométrie de faible valeur [11]. On peut donc les représenter par leur médiane granulométrique $Q_{2}$ qui, pour les sites prévus pour les essais sera comprise entre 150 et $500 \mu \mathrm{m}$.

Dans le cas d'émission à fréquence élevée et d'une répartition granulométrique de forme gaussienne, le coefficient de réverbération varie très rapidement avec l'angle de rasance et croît linéairement avec le logarithme de la fréquence.

On peut donc admettre que la variation de $R_{\mathrm{F}}$ soit représentée par une fonction de la forme :

où :

$$
R_{\mathrm{F}(\mathrm{dB})}=a+b \log F
$$

$a$ représente l'influence de l'angle de rasance (dans ce cas $\phi=45^{\circ}, a=-55 \mathrm{~dB}$ )

$b$ représente la pente de la droite égale à $15,6 \mathrm{~dB}$ (Fig. 3).

La fréquence influe également sur l'atténuation $(\alpha)$ des ondes au cours de leur trajet à cause de différents effets (relaxation ionique, diffusion autour des particules en suspension). On admettra que $\alpha$ croît comme le carré de la fréquence du moins au-delà de $500 \mathrm{kHz}$ [1] (Fig. 4).

Compte tenu des distances parcourues et bien que l'atténuation à ces fréquences soit encore faible, il convient de prendre ce phénomène en considération lors du choix de la fréquence.

Nous avons opté pour la fréquence élevée de $600 \mathrm{kHz}$ qui n'entraîne qu'une faible atténuation dans l'eau de mer $(\alpha<0,1 \mathrm{~dB} / \mathrm{m})$ et qui autorise un index de réverbération compris entre $-10 \mathrm{~dB}$ 


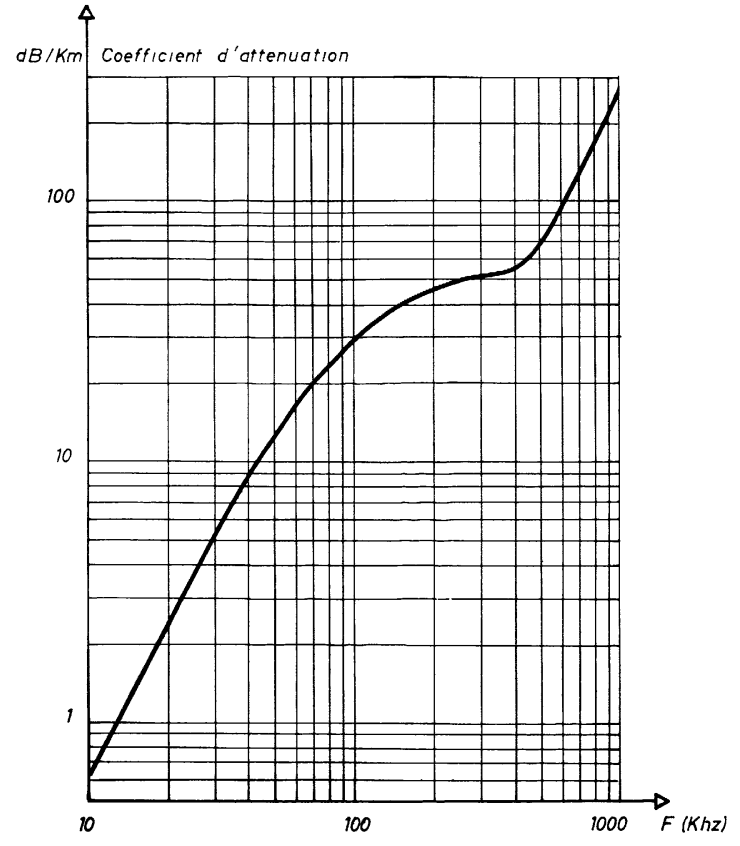

Fig. 3. - Absorption acoustique dans l'eau de mer en fonction de la fréquence (température de l'eau $10^{\circ} \mathrm{C}$-profondeur de l'essai $30 \mathrm{~m}$ ).

[Sound attenuation in sea water versus frequency (sea water temperature $10{ }^{\circ} \mathrm{C}$-measurement depth $30 \mathrm{~m}$ ). ]

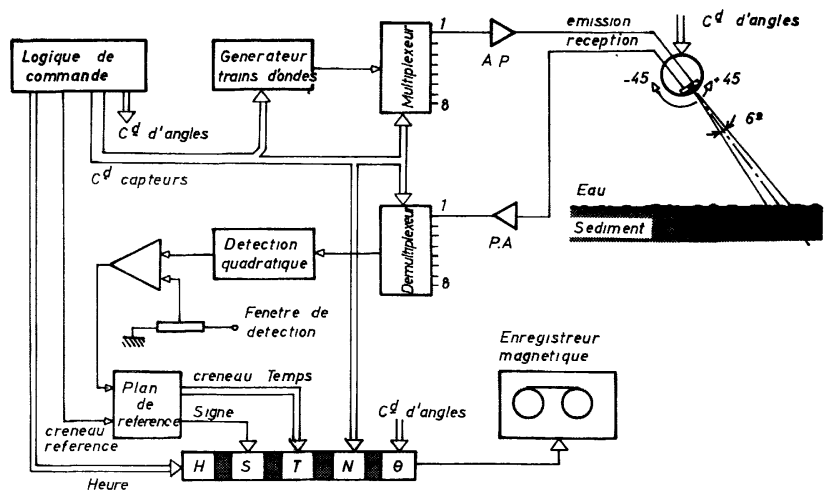

Fig. 4. - Schéma synoptique du micro-topographe.

[Block diagram of the acoustic system.]

et $-15 \mathrm{~dB}$ pour une incidence de $45^{\circ}$ sur un sédiment sableux. Compte tenu de cette fréquence de travail, la durée de l'impulsion est portée à $50 \mu$ s. Dans le cas le plus défavorable (incidence et distance maximum), la différence entre niveau émis et reçu $L-S$ sera de l'ordre de $-60 \mathrm{~dB}$.

Le niveau de l'écho renvoyé par le cylindre est important. L'index de réflexion $T x$ vaut [2] :

où :

$$
T x=10 \log \frac{a L^{2}}{2 \lambda}
$$

$a=$ diamètre du cylindre,

$L=$ longueur soumise aux ultra-sons,

$\lambda=$ longueur d'onde.
Les conditions d'expérience font que $T x$ est proche de $15 \mathrm{~dB}$. Le contraste entre le cylindre et le sédiment $(25 \mathrm{~dB})$ permet une localisation précise de l'obstacle.

Compte tenu des sensibilités des céramiques piézoélectriques utilisées, une impulsion électrique de $20 \mathrm{~V}$ à l'émission provoque après rétrodiffusion sur le fond une tension aux bornes du capteur de réception comprise entre quelques millivolts et $50 \mathrm{mV}$.

\section{3 ELECTRONIQUe DE COMMANDE.}

3.3.1. Généralités. - Les paramètres acoustiques étant imposés par la qualité du sédiment et la géométrie de la zone à explorer, il reste à définir les paramètres de l'électronique de commande et plus particulièrement les fréquences de scrutation.

Le problème consiste à trouver un compromis entre les capacités de stockage de l'information, les réserves énergétiques d'alimentation des différents systèmes électroniques, l'intérêt d'avoir une durée d'observation la plus longue possible et le maximum d'informations par marée.

Les fréquences de variation des phénomènes naturels qui conditionnent le comportement des fonds imposent une période de scrutation inférieure à quelques heures. Nous avons retenu un système permettant de relever une carte toutes les 2 heures.

3.3.2. Principe de fonctionnement. - Pendant chaque séquence de mesure, l'électronique sollicite tour à tour les couples de sondes pour chacune des 16 positions angulaires prises par le tube porte-sonde (Fig. 4). L'utilisation de couple émetteur-récepteur s'est avérée techniquement préférable pour des raisons de couplage électronique.

Un générateur de trains d'ondes excite le transducteur d'émission, lequel génère une vibration acoustique qui après propagation et réflexion sur le fond marin revient en direction de la céramique de réception. La tension résultante aux bornes de cette céramique est amplifiée (gain $=50 \mathrm{~dB}$ ) puis subit une détection quadratique améliorant sensiblement le rapport signal/bruit.

La présence de la fenêtre de détection à seuil variable produit deux effets :

- élimination par filtrage temporel des échos parasites (notamment l'onde directe entre les deux céramiques),

- détection de la présence de l'écho par comparateur.

L'information «temps de transit» de l'impulsion ultrasonore prise par rapport à une référence (voir annexe) est traitée puis enregistrée sur une bande magnétique.

\section{Exploitation des données.}

A chaque point de mesure, à l'information temps de transit de l'impulsion est associé :

- le numéro de micro sondeur sollicité, 
- la valeur de l'angle d'incidence des capteurs,

- la position par rapport au plan de référence,

- l'heure de la mesure.

4. 1 Précision ET PERformances. - Les mesures d'affouillement seront exprimées par rapport à un plan de référence (cf. annexe) et se font en multipliant le retard de l'écho de fond par la célérité des ondes longitudinales dans l'eau.

L'électronique associée à ces mesures de temps permet d'enregistrer une variation maximale de $\pm 1,5 \mathrm{~m}$ par rapport à ce plan de référence défini par les appuis du bâti métallique. La résolution, c'est-à-dire la valeur minimale enregistrable, tient compte des erreurs de détection de l'écho et est estimée à $0,5 \mathrm{~cm}$.

Les erreurs de mesures sont, pour une part mineure, imputables au système de mesures du retard de l'écho soit environ $0,5 \%$ et, pour une part importante, tributaire des variations de célérité acoustique qui règnent au fond lors de la mesure.

La célérité des ondes augmente d'environ $20 \mathrm{~m} / \mathrm{s}$ lorsque la température croît de 5 à $10^{\circ}$, ce qui provoque une erreur relative extrême de $1,3 \%$ [2].

L'erreur maximale commise sur l'évaluation de la hauteur est de $\pm 2 \mathrm{~cm}$

\subsection{RÉSULTATS ET INTERPRÉTATIONS DES DONNÉES. -} Deux séries d'essais ont été réalisées en bordure de côte dans l'Océan Atlantique par une profondeur de $30 \mathrm{~m}$ sur des fonds sableux dont la médiane granulométrique est de $500 \mu \mathrm{m}$ (Fig. 5) et présentant une faible hétérométrie $(S<2)$. Le site et la période d'essais furent choisis en fonction des conditions hydrodynamiques optimums (marnage important d'environ $6,5 \mathrm{~m}$ ).

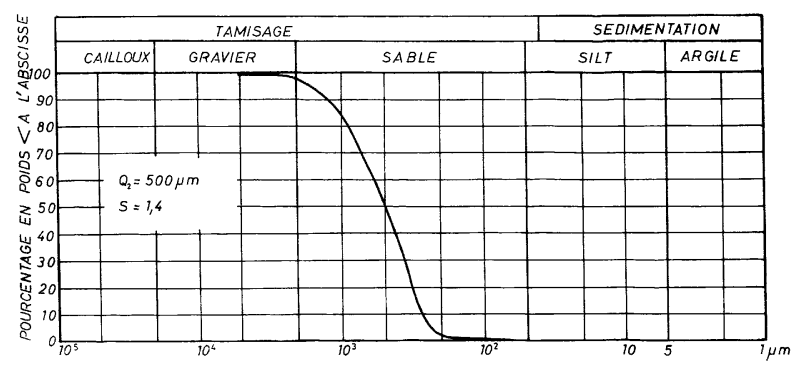

Fig. 5. - Courbe granulométrique cumulée des sédiments du site d'essai.

[Granulometry curve of sediments tested.]

L'exploitation des enregistrements a permis l'obtention d'une série de cartes reflétant l'évolution de la topographie autour de l'obstacle pendant une semaine (Fig. 6).

Nous avons évalué, à partir d'un maillage de la zone à contrôler, l'apport ou l'arrachement de particules sédimentaires de part et d'autre de l'obstacle.
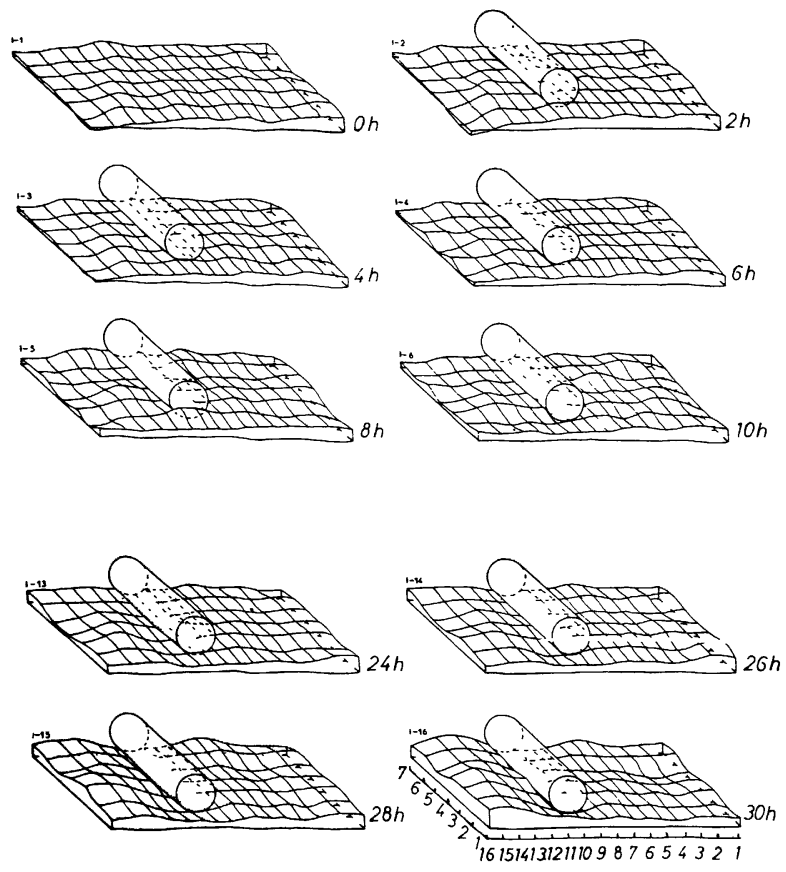

Fig. 6. - Evolution de la topographie autour de l'obstacle. Echantillonnage de résultats relevés in situ.

[Topography variation around target. In situ few data.]

Le calcul de la masse sédimentaire en mouvement tient compte de l'évolution au cours du temps des 128 volumes élémentaires de section connue. L'appauvrissement ou l'enrichissement en sable est déterminé par rapport à la situation en début d'essais :

$$
V=\sum_{i=1}^{128} V_{i}=\sum_{i=1}^{128} S_{i} h_{i}
$$

où $V$ est le volume total arraché ou apporté,

$V_{i}$ est le volume élémentaire,

$h_{i}$ est la hauteur par rapport au plan de référence,

$S_{i}$ est une section élémentaire.

Durant cette période, les courants de direction sensiblement perpendiculaire à l'axe du cylindre ont été de faible amplitude (vitesse maxima de $0,30 \mathrm{~m} / \mathrm{s}$ et l'amplitude de la houle n'a pas dépassé $1 \mathrm{~m}$ alors que la profondeur est de l'ordre de $25 \mathrm{~m}$ ). Ces conditions hydrodynamiques n'ont pas permis de mouvements importants du sable dont la médiane granulométrique est de $500 \mu \mathrm{m}$ (Fig. 5).

Les calculs de transits sédimentaires font apparaître un transport de matériaux d'un côté à l'autre de la zone dans une direction perpendiculaire au cylindre qui coïncide avec la direction privilégiée de la marée.

On obtient ainsi pour le premier essai un arrachement moyen en volume de $0,03 \mathrm{~m}^{3}$ à l'avant de l'obstacle sur une surface de $4 \mathrm{~m}^{2}$.alors que $0,17 \mathrm{~m}^{3}$ de sable sur une surface de $4 \mathrm{~m}^{2}$ également sont apportés à l'arrière du cylindre. Le bilan en fin d'essai 
s'avère positif, ce transit sédimentaire provient d'une zone située en amont de l'objet et est le résultat de la présence du corps mouillé.

Ces valeurs restent comparables à celles correspondant à un charriage sédimentaire effectué en l'absence d'un obstacle. Un apport de $0,04 \mathrm{~m}^{3}$ par $\mathrm{m}^{2}$ correspond à ce que nous aurions calculé pour un sable dans les mêmes conditions $\left(0,07 \mathrm{~m}^{3}\right.$ par $\mathrm{m}^{2}$ [12]) pour des courants beaucoup plus forts. Il semble donc, dans cette phase de l'étude, que le corps ait eu deux actions :

- une augmentation des champs de courant à proximité de l'obstacle,

- une perturbation des mouvements quasi alternatifs des sables en transits dans la zone considérée.

Le phénomène d'apport et d'arrachement de particules sédimentaires apparaît nettement sur la figure 7

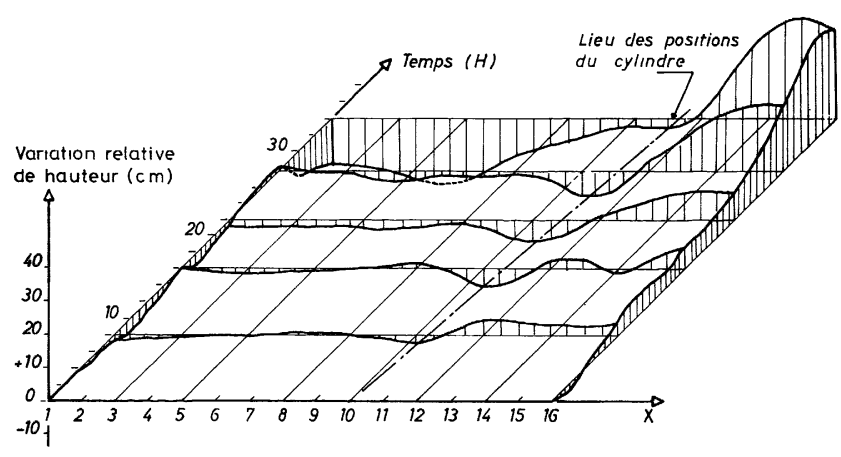

Fig. 7. - Evolution du profil moyen en fonction du temps.

[Mean contour evolution versus time.]

où sont portés, en fonction du temps, les profils moyennés (suivant la direction parallèle à l'axe du cylindre) le profil initial (au temps 0 ) étant pris comme référence. On remarquera le glissement de l'empreinte du cylindre en fonction du temps. En effet, des mesures complémentaires corroborent ce résultat et indiquent une rotation du cylindre de quelques degrés.

\section{Conclusion.}

Les premières campagnes de mesures organisées montrent le bon fonctionnement du système proposé. Le dispositif ne perturbe pas les conditions hydro- dynamiques sur le site expérimental et on peut avoir une bonne estimation globale des transits sédimentaires à la fois en quantité et en direction. Les résultats montrent que le principe de la mesure acoustique est adapté à ce genre de situation et que la précision des mesures est suffisante.

Une étude qui doit aboutir à un modèle mathématique déterminé à partir de données expérimentales est en cours.

\section{Annexe}

\section{Plan de référence.}

La mesure de hauteur d'affouillement se fait en comparant le temps que met l'écho renvoyé par le fond réel à un écho de référence que renverrait un fond idéal plat. Le temps de transit de l'écho référence est pondéré en fonction de l'angle d'incidence du faisceau acoustique et de la hauteur des sondes par rapport à ce fond plat.

Ainsi, lors du balayage du faisceau acoustique, à chaque position angulaire l'écho de fond sera comparé à un écho de référence différent. L'écart entre ces deux signaux donne une information signée (positif si l'écho de fond arrive avant l'écho référence ceci dans le cas d'un remblais de sédiment, négatif pour le cas inverse, c'est-à-dire d'un appauvrissement en sédiment).

\section{Ancrage du bâti.}

L'exactitude des mesures dépend de l'immobilité du dispositif de mesure acoustique dans l'espace pendant l'expérience. La remise en suspension et le transit du sédiment ne concernent qu'une épaisseur relativement faible d'environ $30 \mathrm{~cm}$ [12]. Il est donc important d'ancrer le bâti sur un substrat qui est fixe. Les deux tripodes qui soutiennent le cylindre portesonde, comportent chacun trois tarières enfoncées dans le sol. Leur forme assure un ancrage efficace; en effet les vis hélicoïdales s'enfoncent de plus de $90 \mathrm{~cm}$ sous l'interface eau-sédiment et ont une résistance à l'arrachement de $3000 \mathrm{~N}$.

Les tarières parfaitement fixées à cette profondeur assurent un plan de référence indépendant de la dynamique sédimentaire.

\section{Bibliographie}

[1] Caruthers, J. W., Fundamentals of Marine Acoustics, (ElSeVIER OCEanography SerIE 18) 1977.

[2] Pasteau, H., Théorie et applications de l'acoustique sous-marine, Tome II, E.N.S.T.A., 1973.

[3] Waton, G., Candau, S., Revue sur les mesures d'absorption acoustique d'ondes sonores de basse fréquence dans l'eau de mer, Rev. Cethedec 16e année, 67 (1981).
[4] Harold, F., Messias, Bathymetric system Performance Prediction. IEE Ocean (1975).

[5] Le Gall, J., Mesure des index monostatiques et bistatiques de réverbération de fond et de réflexion d'objets divers., Rev. Cethedec (ondes et signal) 72 (1982).

[6] Welton, P. J., Forward and Specular scattering from randomly rough surfaces, Rev. Cethedec 60 (1979). 
[7] Welton, P. J., Backscattering from randomly rough surfaces, Rev. Cethedec 60 (1979).

[8] Gazanhes, C., Quelques aspects de la réverbération de surface, Rev. Cethedec 48 (1976).

[9] Clavellous, Etude de la réverbération, Rev. Cethedec 44 (1975).

[10] Longuemard, J. P., Mesure in situ de l'amplitude des ondes réfléchies ou rétrodiffusées par les sédiments marins. Application à la détermination in situ des modes grunulométriques, Revue Phys. Appl. 13 (1978).

[11] Bonnefille, Cours d'hydraulique maritime, Collection ENSTA (Masson) 1976.

[12] Longuemard, J. P., Sur une nouvelle méthode d'étude des transits sédimentaires sur les plateaux continentaux. Symposium International RSEEPC. Juillet 1974. 